Paper

\title{
A simple numerical verification method for differential equations based on infinite dimensional sequential iteration
}

\author{
Yoshitaka Watanabe ${ }^{1 a)}$ \\ ${ }^{1}$ Research Institute for Information Technology, Kyushu University \\ 6-10-1 Hakozaki, Higashi-ku, Fukuoka 812-8581, Japan \\ a)watanabe@cc.kyushu-u.ac.jp
}

Received April 10, 2012; Revised July 26, 2012; Published January 1, 2013

\begin{abstract}
This paper describes a numerical verification of solutions for infinite dimensional functional equations based on residual form and sequential iteration. Comparing with other verification procedures as typified by Newton-type iterations, the proposed algorithm can be done at low computational cost, although it needs that the formulated compact map is retractive in some neighborhood of the fixed-point to be verified. Several computer-assisted proofs for differential equations, including nonlinear partial differential equations will be shown.
\end{abstract}

Key Words: computer-assisted proof, functional equations, differential equations, residual form, sequential iteration

\section{Functional equations and residual form}

This section introduces general functional equations and two kind of residual forms by using approximate solutions.

\subsection{Problem}

Let $\hat{X}$ be the Banach space and $X, Y$ the Hilbert spaces satisfying $\hat{X} \hookrightarrow X \hookrightarrow Y$ with the compactness of the embedding $\hat{X} \hookrightarrow X$. Let $\mathcal{A}$ be a linear operator from $\hat{X}$ to $Y$, and $f$ a (generally nonlinear) operator from $X$ to $Y$. Consider the problem to find $u$ which satisfies

$$
\mathcal{A} u=f(u) .
$$

For nonlinear differential equations, in Eq. (1), the operator $\mathcal{A}$ stands for the linear map including the highest order differential term and $f$ the other nonlinear term (see Section 3). Define the inner products and norms of the Hilbert spaces $X$ and $Y$ by $(u, v)_{X},(u, v)_{Y}$ and $\|u\|_{X}=\sqrt{(u, u)_{X}}$, $\|u\|_{Y}=\sqrt{(u, u)_{Y}}$, respectively. Note that when applying "direct" residual formulation described in Section 1.2.1, it is possible to set $X$ and $Y$ as the Banach spaces because the inner product is not necessarily required.

Suppose that the operators $\mathcal{A}$ and $f$ have the following:

A1. For each $\phi \in Y, \mathcal{A} \psi=\phi$ has the unique solution $\psi \in \hat{X}$ and this mapping $\phi \mapsto \psi$ is continuous. 
By defining the mapping of $\mathbf{A} \mathbf{1}$ by $\psi=\mathcal{A}^{-1} \phi$, the operator $\mathcal{A}^{-1}: Y \rightarrow X$ becomes compact due to the compactness of the embedding $\hat{X} \hookrightarrow X$. Note that $\mathcal{A}^{-1}$ can be defined as the composite mapping of the continuous operaror $\mathscr{A}^{-1}: Y \rightarrow \hat{X}$ (usually the inverse of $\mathcal{A}$ ) and the embedding operator $I_{\hat{X} \hookrightarrow X}$ as follows.

$$
\mathcal{A}^{-1}:\left\{\begin{array}{ccccc}
Y & \underset{\mathscr{A}^{-1}}{\text { cont. }} & \hat{X} & \frac{I_{\hat{X} \hookrightarrow X}}{\text { compact }} & X \\
\phi & \mapsto & \psi & \mapsto & \psi
\end{array}\right.
$$

A2. There exists a constant $C_{p}>0$ satisfying

$$
\left\|\mathcal{A}^{-1} \phi\right\|_{X} \leq C_{p}\|\phi\|_{Y}, \quad \forall \phi \in Y .
$$

In the actual verified computation, an explicit value of $C_{p}$ has to be evaluated.

A3. $f: X \rightarrow Y$ is continuous and maps bounded sets in $X$ into bounded sets in $Y$.

\subsection{Residual forms}

Using a fixed approximate solution $u_{h} \in X$ for problem (1), we consider two types of residual equations.

\subsubsection{Direct residual form}

When the approximate solution $u_{h} \in X$ satisfies $\mathcal{A} u_{h} \in Y$, Eq. (1) can be rewritten as

$$
\mathcal{A} w=f\left(w+u_{h}\right)-\mathcal{A} u_{h}
$$

to find the residual

$$
w:=u-u_{h}
$$

Setting the right hand side of Eq. (3) by $g(w): X \rightarrow Y$, we obtain the residual equation $\mathcal{A} w=g(w)$. It is expected that if $u_{h}$ is an accurate approximation of $u$, the residual term $g$ will be small.

\subsection{2 $X^{*}$-type residual form}

In the case of $\mathcal{A} u_{h}$ does not belong to $Y$, we apply the following " $X^{*}$-type" residual formulation [1]. " $X^{*}$ " means the dual space of $X$. Let $X_{h}$ be a finite dimensional approximation subspace of $X$ depending on the parameter $h>0$. For example, $X_{h}$ is taken to be a finite element subspace with mesh size $h$. Define the orthogonal projection $P_{h}: X \rightarrow X_{h}$ by

$$
\left(v-P_{h} v, v_{h}\right)_{X}=0, \quad \forall v_{h} \in X_{h},
$$

and suppose that $P_{h}$ has the following approximation property.

$$
\left\|\left(I-P_{h}\right) v\right\|_{X} \leq C(h)\|\mathcal{A} v\|_{Y}, \quad \forall v \in \hat{X} .
$$

Here, $C(h)$ is a positive constant which is numerically determined with the property that $C(h) \rightarrow 0$ as $h \rightarrow 0$. We also suppose that $\mathcal{A}: \hat{X} \rightarrow Y$ satisfies

$$
(u, v)_{X}=(\mathcal{A} u, v)_{Y}, \quad \forall u \in \hat{X}, \quad \forall v \in X .
$$

From Eq. (5), problem (1) is rewritten in the weak form: find $u \in X$ such that

$$
(u, v)_{X}=(f(u), v)_{Y}, \quad \forall v \in X .
$$

Moreover, from Eq. (4) and A1, Aubin-Nitsche's trick [2] derives

$$
\left\|\left(I-P_{h}\right) v\right\|_{Y} \leq C(h)\left\|\left(I-P_{h}\right) v\right\|_{X}, \quad \forall v \in X .
$$

Now assume that the approximate solution $u_{h} \in X_{h}$ satisfies 


$$
\left(u_{h}, v_{h}\right)_{X}=\left(f\left(u_{h}\right), v_{h}\right)_{Y}, \quad \forall v_{h} \in X_{h}
$$

exactly. It is rather natural for our situation of the verification to assume the existence of the such approximate solution since it is a finite dimensional problem. In general, it is difficult to take the approximate solution $u_{h}$ satisfying Eq. (7) in a mathematically rigorous sense. However, it is not so difficult that we strictly enclose the solution by validated computations for a finite dimensional nonlinear problem with interval arithmetic [3, Section 13], [4, function verifynlss].

From $\mathbf{A} 1$, for each $f\left(u_{h}\right) \in Y$, there exists $\bar{u} \in X$ such that

$$
\mathcal{A} \bar{u}=f\left(u_{h}\right) .
$$

Thus by using Eq. (5) it holds that

$$
(\bar{u}, v)_{X}=\left(f\left(u_{h}\right), v\right)_{Y}, \quad \forall v \in X,
$$

and by restricting $v \in X$ of Eq. (8) to the element of $X_{h}$ and substituting Eq. (7),

$$
\left(\bar{u}-u_{h}, v_{h}\right)_{X}=0, \quad \forall v_{h} \in X_{h}
$$

is implied. Eq. (9) means that $P_{h} \bar{u}$ coincide with $u_{h}$. Here, setting

$$
v_{0}:=\bar{u}-u_{h},
$$

and using Eqs. (4) and (6), we have the following norm estimations:

$$
\left\|v_{0}\right\|_{X} \leq C(h)\left\|f\left(u_{h}\right)\right\|_{Y}, \quad\left\|v_{0}\right\|_{Y} \leq C(h)^{2}\left\|f\left(u_{h}\right)\right\|_{Y} .
$$

Finally, setting $w=u-\bar{u}$, we obtain an another residual form for $w$ instead of Eq. (3):

$$
\mathcal{A} w=f\left(w+u_{h}+v_{0}\right)-f\left(u_{h}\right) .
$$

Therefore similarly to Section 1.2.1 we have the residual equation $\mathcal{A} w=g(w)$ setting $g(w):=f(w+$ $\left.u_{h}+v_{0}\right)-f\left(u_{h}\right)$ and if we obtain the solution $w \in X$ of Eq. (10), we can also construct the solution $u$ of Eq. (1) by

$$
u=u_{h}+v_{0}+w .
$$

Note that this residual form (10) does not require $\mathcal{A} u_{h} \in Y$ and, for concrete operators $\mathcal{A}$, it can be possible to present a posteriori estimates of $v_{0}$ with higher order finite element basis of $X$ [57]. Moreover, under the assumption (5), constant $C_{p}$ satisfying Eq. (2) can be taken the embedding constant $X \hookrightarrow Y$ such that

$$
\|\psi\|_{Y} \leq C_{p}\|\psi\|_{X}, \quad \forall \psi \in X
$$

because

$$
\begin{aligned}
\left\|\mathcal{A}^{-1} \phi\right\|_{X}^{2} & =\left(\mathcal{A}^{-1} \phi, \mathcal{A}^{-1} \phi\right)_{X}=\left(\phi, \mathcal{A}^{-1} \phi\right)_{Y} \\
& \leq\|\phi\|_{Y}\left\|\mathcal{A}^{-1} \phi\right\|_{Y} \\
& \leq C_{p}\|\phi\|_{Y}\left\|\mathcal{A}^{-1} \phi\right\|_{X}
\end{aligned}
$$

by the Schwarz inequality.

\section{Verification algorithm IS-Res}

This section is devoted a verification algorithm IS-Res ${ }^{1}$ based on infinite dimensional sequential iterations.

${ }^{1}$ It comes from "Infinite", "Sequential", and "Residual." 


\subsection{Fixed-point form}

Consider the residual form described in Section 1.2:

$$
\mathcal{A} w=g(w),
$$

and aim to verify the existence of $w \in X$ around 0 . Since $g: X \rightarrow Y$ satisfies the same condition A3 as $f$, denoting compounded operator $F$ by

$$
F:=\mathcal{A}^{-1} \circ g: \quad X \rightarrow X,
$$

by virtue of A1, problem (1) can be rewritten as the fixed-point equation to find $w \in X$ such that

$$
w=F(w) .
$$

From $\mathbf{A} \mathbf{3}$ and the compactness of $\mathcal{A}^{-1}: Y \rightarrow X, F$ is also the compact map on $X$ as follows:

$$
F:\left\{\begin{array}{ccccc}
X & \frac{g}{\longrightarrow} & Y & \underset{\mathcal{A}^{-1}}{\longmapsto} & X \\
w & \stackrel{\text { cont. bdd. }}{\mapsto} & g(w) & \mapsto & \mathcal{A}^{-1} \circ g(w)
\end{array} .\right.
$$

\subsection{Verification condition}

The following verification theorem is derived by condition that the operator $F$ is retractive in some neighborhood of zero.

Theorem 1 For $\alpha>0$, set a candidate set $W \subset X$, which is expected to enclose a fixed-point, by

$$
W:=\left\{w \in X \mid\|w\|_{X} \leq \alpha\right\},
$$

then

$$
C_{p} \sup _{w \in W}\|g(w)\|_{Y} \leq \alpha
$$

implies that $F$ has a fixed-point in $W$.

Proof. Since $W \subset X$ is the nonemply, bounded, convex, and closed set centered at zero, by using Eqs. (2), (13),

$$
\sup _{w \in W}\|F(w)\|_{X} \leq C_{p} \sup _{w \in W}\|g(w)\|_{Y} \leq \alpha
$$

implies $F(W) \subset W$. Theorefore the Schauder fixed-point theorem [8, Theorem 2.A] asserts the conclusion.

\subsection{Local uniqueness of the solution}

Theorem 2 below assures the local uniqueness of the solution in the candidate set $W$.

Theorem 2 For the candidate set $W \subset X$ defined by Eq. (12), assume that there exists $C_{g}>0$ such that

$$
\left\|g\left(w_{1}\right)-g\left(w_{2}\right)\right\|_{Y} \leq C_{g}\left\|w_{1}-w_{2}\right\|_{X}, \quad \forall w_{1}, w_{2} \in W .
$$

Then adding to the condition (13) for Theorem 1, if

$$
C_{p} C_{g}<1
$$

holds, the fixed-point of $F$ is unique in $W$.

Proof. The set $W$ is nonemply closed, and it is true that

$$
\begin{aligned}
\left\|F\left(w_{1}\right)-F\left(w_{2}\right)\right\|_{X} & =\left\|\mathcal{A}^{-1}\left(g\left(w_{1}\right)-g\left(w_{2}\right)\right)\right\|_{X} \\
& \leq C_{p} C_{g}\left\|w_{1}-w_{2}\right\|_{X}, \quad \forall w_{1}, w_{2} \in W
\end{aligned}
$$

from Eqs. (2), (14). Therefore, the condition (15) implies that $F$ is a contractive map. Thus the Banach fixed-point theorem [8, Theorem 1.A] implies the local uniqueness.

Moreover, when the map $g$ is differentiable on $W$, we obtain a condition for computing $C_{g}$. 
Corollary 1 For the same candidate set $W \subset X$ as in Theorem 2, if $g$ is Fréchet differentiable on $W$, then the constant $C_{g}>0$ for Eq. (14) can be taken to satisfy

$$
\sup _{\hat{w} \in W}\left\|g^{\prime}[\hat{w}] w\right\|_{Y} \leq C_{g}\|w\|_{X}, \quad \forall w \in X .
$$

Proof. Since $W$ is convex set, the mean value theorem [9, Lemma 2.3] shows

$$
\left\|g\left(w_{1}\right)-g\left(w_{2}\right)\right\|_{Y} \leq \sup _{\hat{w} \in W}\left\|g^{\prime}[\hat{w}]\left(w_{1}-w_{2}\right)\right\|_{Y}, \quad \forall w_{1}, w_{2} \in W .
$$

Then by setting $w=w_{1}-w_{2} \in X$, Eq. (16) asserts Eq. (14).

Remark 2.1 From Eq. (17) the boundedness (16) on $\hat{w} \in W$ for the linear operator $g^{\prime}[\hat{w}]: X \rightarrow Y$ can be replaced to "milder" condition for $W$ :

$$
\sup _{\hat{w} \in W}\left\|g^{\prime}[\hat{w}]\left(w_{1}-w_{2}\right)\right\|_{Y} \leq C_{g}\left\|w_{1}-w_{2}\right\|_{X}, \quad \forall w_{1}, w_{2} \in W .
$$

Remark 2.2 Let us apply $\mathcal{A}^{-1}$ to the problem (1) and consider the fixed-point equation $u=\mathcal{A}^{-1}$ 。 $f(u)$ directly. Then for the approximate solution $u_{h}$ and $W$ defined by Eq. (12), we set the candidate set $U$ as $U=u_{h}+W$ and check the condition for the Schauder fixed-point theorem by

$$
\mathcal{A}^{-1} \circ f(U)-u_{h} \subset W \text {. }
$$

However, in order to apply $\mathbf{A 2}$ (2), it has to be

$$
\mathcal{A}^{-1} \circ f(u)-u_{h}=\mathcal{A}^{-1} \circ\left(f(u)-\mathcal{A} u_{h}\right), \quad \forall u \in U,
$$

therefore, $\mathcal{A} u_{h} \in Y$ is required. Consequently, when $A u_{h} \notin Y$, the residual formulation is indispensable.

\subsection{Verification algorithm IS-Res}

Based on Theorems 1 and 2, we give the following verification algorithm IS-Res. The extension procedure involving $\varepsilon$ occurring in this algorithm is called " $\varepsilon$-inflation" which is a kind of acceleration technique [10]. The concrete value of $\varepsilon>0$ should be adapted to the actual problem.

\section{Verification algorithm IS-Res}

- $k=0$

Set an initial value $\alpha^{(0)}>0$.

- $k \geq 1$

1. For a fixed small constant $\varepsilon>0$ set

$$
\hat{\alpha}^{(k)}:=(1+\varepsilon) \alpha^{(k-1)} .
$$

2. The $k$-th candidate set $W^{(k)}$ is defined by

$$
W^{(k)}:=\left\{w \in X \mid\|w\|_{X} \leq \hat{\alpha}^{(k)}\right\} .
$$

3. Compute $k$-th iteration by

$$
\alpha^{(k)}:=C_{p} \sup _{w \in W^{(k)}}\|g(w)\|_{Y} .
$$

4. If $\alpha^{(k)} \leq \hat{\alpha}^{(k)}$ then stop, and there exists a desired solution in $W^{(k)} \subset X$. Moreover for $W^{(k)}$, if $C_{p} C_{g}<1$ holds then the solution is unique within $W^{(k)}$ (There is an optional extra to generate $\hat{W}$ in Corollary 2.).

5. Setting $k:=k+1$ and return to the step 1 . If $k$ reaches a maximum iteration number or $\alpha^{(k)}$ exceeds a criterion then stop, and the verification fails. 
Remark 2.3 In the verification algorithm IS-Res, since assuming the constant $C_{p}$ is known, the main computational cost concentrates on the estimation of the norm $\sup _{w \in W}\|g(w)\|_{Y}$ for the candidate set $W$ and the estimation $C_{g}$ for the local uniqueness. In another verification approaches, such as Newton-type methods, some "heavy" computations, for example, bounds for inverse operator of linearized problems for Eq. (1) [11-14] or verified computations for interval linear systems [15] are needed. One of peculiar features of IS-Res is its low cost since it can avoid such computations (see Section 3.2).

\subsection{Extension of the area for local uniqueness}

From the point of view of the principle: "To aim narrower enclosure area and wider uniqueness area," constant $C_{g}$ satisfying the condition (15) should be taken as larger as possible. The following is one of extension procedures for the candidate set assuring local uniqueness.

Corollary 2 Suppose that the candidate set $W \subset X$ defined by Eq. (12) has a fixed-point $w$ of $F$. If a set

$$
\hat{W}:=\left\{w \in X \mid\|w\|_{X} \leq \hat{\alpha}\right\}
$$

for $\hat{\alpha} \geq \alpha>0$, there exists $\hat{C}_{g}>0$ such that

$$
\left\|g\left(w_{1}\right)-g\left(w_{2}\right)\right\|_{Y} \leq \hat{C}_{g}\left\|w_{1}-w_{2}\right\|_{X}, \quad \forall w_{1}, w_{2} \in \hat{W},
$$

and $C_{p} \hat{C}_{g}<1$, then the fixed-point of $F$ is unique within $\hat{W}$.

Proof. Assume that there exists fixed-point $\hat{w} \in \hat{W}$ of $F$ such that $\hat{w} \neq w$, then from Eqs. (2), (18) we have

$$
\begin{aligned}
\|w-\hat{w}\|_{X} & =\|F(w)-F(\hat{w})\|_{X}=\left\|\mathcal{A}^{-1} \circ(g(w)-g(\hat{w}))\right\|_{X} \\
& \leq C_{p} \hat{C}_{g}\|w-\hat{w}\|_{X} .
\end{aligned}
$$

By dividing the both sides by $\|w-\hat{w}\|_{X} \neq 0, C_{p} \hat{C}_{g} \geq 1$ holds. However it contradicts with $C_{p} \hat{C}_{g}<1$, therefore the fixed-point is only $w$.

Using Corollary 2 we can obtain nonexistence proof for the area $\hat{W} \backslash W$. The extension procedure $\varepsilon$-inflation can also be applied to generate $\hat{W}$ at the initial value $\alpha$ [16, Section 7.11.6].

\section{Verification results}

This section shows some computer-assisted proofs of solutions for differential equations by IS-Res, and considers its effectiveness and limitation. In the actual computation, we have to treat the infinite dimensional term. Moreover, it is impossible to avoid the effect of rounding error of floating-point arithmetic. However, by norm estimates and interval arithmetic software taking into account effects of rounding error, we can obtain mathematically rigorous upper bounds for $\alpha^{(k)}$ in the over-estimated sense. We use the interval arithmetic toolbox INTLAB [4] Version 6 with MATLAB R2012a.

For some integer $m$, let $H^{m}(\Omega)$ denote the $L^{2}$-Sobolev space of order $m$ on bounded domain $\Omega$. We define

$$
H_{0}^{1}(\Omega):=\left\{u(x) \in H^{1}(\Omega) \mid u(x)=0, x \in \partial \Omega\right\}
$$

with the inner product $(\nabla u, \nabla v)_{L^{2}}$ and the norm $\|u\|_{H_{0}^{1}(\Omega)}:=\|\nabla u\|_{L^{2}(\Omega)}$, where

$$
\nabla u=\left[\frac{\partial u}{\partial x_{1}}, \ldots, \frac{\partial u}{\partial x_{n}}\right]^{T}
$$

and $(u, v)_{L^{2}}$ stands for $L^{2}$-inner product on $\Omega$. 


\subsection{Two-point boundary value problem}

For $K>0$, consider the following two-point boundary value problem.

$$
\left\{\begin{aligned}
-u^{\prime \prime}-K u= & \left(\pi-\frac{K}{\pi}\right) \sin \pi x, \quad 0<x<1, \\
& u(0)=u(1)=0 .
\end{aligned}\right.
$$

Eq. (19) has the exact solution

$$
u(x)=\frac{1}{\pi} \sin \pi x .
$$

Setting $\mathcal{A} u=-u^{\prime \prime}$, we give function spaces for $\Omega=(0,1)$ by

$$
\hat{X}=H^{2}(\Omega) \cap H_{0}^{1}(\Omega), \quad X=H_{0}^{1}(\Omega), \quad Y=L^{2}(\Omega),
$$

with inner product

$$
(u, v)_{X}=\left(u^{\prime}, v^{\prime}\right)_{L^{2}}, \quad(u, v)_{Y}=(u, v)_{L^{2}},
$$

and apply the verification algorithm IS-Res. We divide the interval $(0,1)$ into $N$ equal parts and take the finite element subspace $X_{h}$ whose elements are piecewise linear polynomials on each subinterval.

The constant $C(h)$ of Eq. (4) can be taken as $h / \pi$ with $h:=1 / N[17]$ and Eq. (2) (Eq. (11)) holds for $C_{p}=1 / \pi[18$, Theorem 1.2$]$.

Due to the approximate solution $u_{h} \in X_{h}$ is $\mathcal{A} u_{h} \notin Y$, we apply $X^{*}$-type residual form in Section 1.2.2. The approximate solution $u_{h} \in X_{h}$ which satisfies the finite dimensional equation (7) can be obtained by solving interval linear system with validated error bounds. Now we can show that, if $K<\pi^{2}$, the condition (13) is hold for $\alpha>0$ such that

$$
\left\|v_{0}\right\|_{L^{2}(\Omega)} \leq \frac{\pi}{K}\left(1-\frac{K}{\pi^{2}}\right) \alpha
$$

This fact suggests that when $K \geq \pi^{2}$ it is difficult to verify the solution by IS-Res and, even if the equation is linear, Newton-type verification techniques [15] are needed. Table I shows upper bounds of norms for $W$ satisfying the condition (13) for $N$ and $K$.

Table I. Verification results (Two-point BVP).

\begin{tabular}{||c|c|c|c||}
\hline$N$ & $K=\pi$ & $K=\pi^{2} / 2$ & $K=99 / 100 \pi^{2}$ \\
\hline 10 & $3.28921 \times 10^{-3}$ & $7.01365 \times 10^{-3}$ & $3.88862 \times 10^{-1}$ \\
20 & $8.24655 \times 10^{-4}$ & $1.76415 \times 10^{-3}$ & $1.46659 \times 10^{-1}$ \\
40 & $2.06312 \times 10^{-4}$ & $4.41715 \times 10^{-4}$ & $4.20342 \times 10^{-2}$ \\
\hline
\end{tabular}

\subsection{Second-order elliptic boundary value problem}

Next we consider the following second-order semilinear elliptic boundary value problems on the unit square $\Omega=(0,1) \times(0,1)$.

$$
\left\{\begin{aligned}
-\Delta u & =\lambda\left(1+u+u^{2}-a u^{3}\right) & & \text { in } \Omega \\
u & =0 & & \text { on } \partial \Omega .
\end{aligned}\right.
$$

Here $\lambda, a>0$ are given parameters. It is well known that, for a small $a$, problem (20) draws a bifurcation diagram parameterized $\lambda$ which is shown in Fig. 1 [19]. We define the partition of $\Omega$ by the linear and uniform triangular meshes with the partition size $h>0$ and construct the $X_{h}$. We set $\mathcal{A}=-\Delta$, and $\hat{X}, X$ and $Y$ are the same as in Section 3.1 for $\Omega=(0,1) \times(0,1)$. The inner product of $X$ becomes $(u, v)_{X}=(\nabla u, \nabla u)_{L^{2}}$. Eq. (4) is held as $C(h)=0.493 h$ [20] and $C_{p}$ of Eq. (2) (Eq. (11)) can be taken $1 /(\pi \sqrt{2})$. We also obtain the approximate solution $u_{h} \in X_{h}$ for Eq. (7) by Krawczyk method solving finite dimensional nonlinear equations with guaranteed accuracy [3, Theorem 13.3]. Figure 2 shows shapes of approximate solutions. They are all "lower solutions" in 


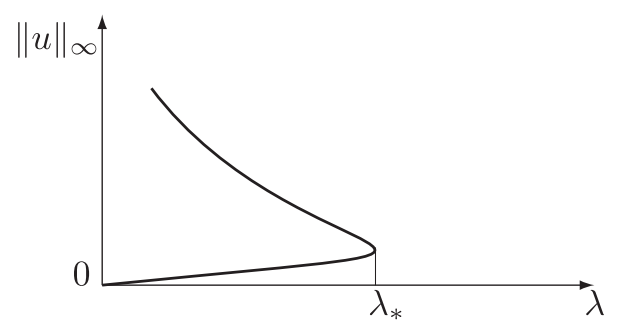

Fig. 1. Bifurcation diagram with $\lambda$ as parameter.

Table II. Verification results $(h=1 / 30, a=0.001)$.

\begin{tabular}{||c|r|r|r|c|r||}
\hline$\lambda$ & $\left\|u_{h}\right\|_{\infty}$ & $\left\|v_{0}\right\|_{H_{0}^{1}(\Omega)}$ & its. & $\|W\|_{H_{0}^{1}(\Omega)}$ & $\|\hat{W}\|_{H_{0}^{1}(\Omega)}$ \\
\hline \hline 1.0 & 0.07816 & $1.70758 \times 10^{-2}$ & 3 & $8.16807 \times 10^{-5}$ & 6.25829 \\
\hline 2.0 & 0.16802 & $3.57832 \times 10^{-2}$ & 4 & $4.48135 \times 10^{-4}$ & 2.88094 \\
\hline 3.0 & 0.27554 & $5.69294 \times 10^{-2}$ & 5 & $1.45700 \times 10^{-3}$ & 1.68467 \\
\hline 4.0 & 0.41234 & $8.21144 \times 10^{-2}$ & 6 & $4.15696 \times 10^{-3}$ & 1.04604 \\
\hline 5.0 & 0.60729 & $1.15381 \times 10^{-1}$ & 9 & $1.37587 \times 10^{-2}$ & 0.51463 \\
\hline 5.5 & 0.75339 & $1.38860 \times 10^{-1}$ & 14 & $3.43893 \times 10^{-2}$ & 0.30793 \\
\hline 6.0 & 0.99555 & $1.76076 \times 10^{-1}$ & - & failed & - \\
\hline
\end{tabular}

$\lambda=1$

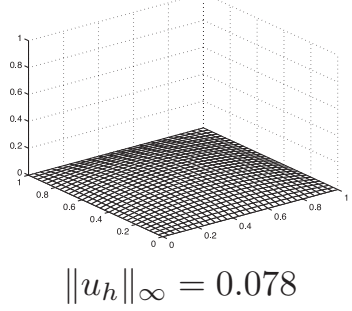

$\lambda=3$

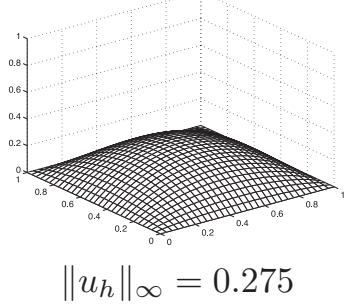

$\lambda=6$

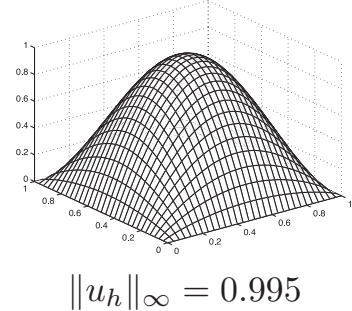

Fig. 2. Shape of approximate solutions (normalized by 1).

the bifurcation diagram (Fig. 1). Table II indicates verification results. $\|W\|_{H_{0}^{1}(\Omega)}$ stands for the upper bound of enclosing the solutions and $\|\hat{W}\|_{H_{0}^{1}(\Omega)}$ the local uniqueness. For detailed calculation for $\sup _{w \in W}\|g(w)\|_{Y}, C_{g}$ and $\hat{C}_{g}$ with MATLAB/INTLAB code, see [16, Section 7.11]. By using IS-Res, we failed verification of "lower solutions" for $\lambda$ exceeding 6 or all "upper solutions." In order to enclose such solutions, we should apply other verification algorithms [14].

We compare computational costs with the existing verification algorithms FN-Int and IN-Linz [14] on Intel Core 2 Duo prosessor $(2.66 \mathrm{GHz})$ for $N=30, a=0.001$ and $\lambda=5.5$. The common elapsed time to obtain an enclosure of $u_{h}$ satisfying Eq. (7) is $25.91 \mathrm{sec}$. The elapsed times of verification are 33.48 sec. (IS-Res), 316.85 sec. (FN-Int), and 45.08 sec. (IN-Linz), respectively.

\subsection{Forth-order elliptic problem}

Consider the following forth-order nonlinear problem on the domain $\Omega=(0, \pi / a) \times(0, \pi)$.

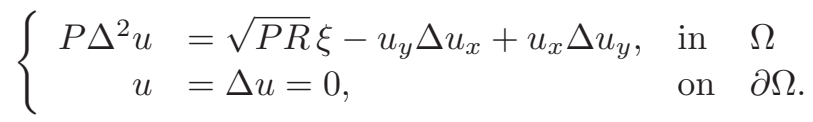

Let constants $P, R, a>0$ be given, $u_{x}=\partial u / \partial x, u_{y}=\partial u / \partial y$, and $\xi$ be an outer force satisfying the same boundary conditions for $u$. The problem (21) comes from two-dimensional Navier-Stokes equations by replacing the velocity field to stream functions and by cross-differentiating the equation of motion to eliminate the pressure [21]. We define the function space by double Fourier series:

$$
X^{k}:=\left\{\sum_{\substack{m=1 \\ n=1}}^{\infty} a_{m n} \sin (a m x) \sin (n y) \mid a_{m n} \in \mathbb{R}, \sum_{\substack{m=1 \\ n=1}}^{\infty}\left((a m)^{2 k}+n^{2 k}\right) a_{m n}^{2}<\infty\right\},
$$


and choose

$$
\mathcal{A}=P \Delta^{2}, \quad \hat{X}=X^{4}, \quad X=X^{3}, \quad Y=X^{0} .
$$

Then for $\|u\|_{X}:=\|\nabla \Delta u\|_{L^{2}(\Omega)},\|u\|_{Y}:=\|u\|_{L^{2}(\Omega)}, \mathbf{A 2}(2)$ holds for $C_{p}=1 /\left(P\left(\sqrt{a^{2}+1}\right)\right)$ and we can apply IS-Res. We set $\xi$ as the approximate solution of the Oberbeck-Boussinesq equation which is the coupled-equation with Eq. (21) and convection diffusion equation [22]. We also set $X_{h}$ as the $N$-th truncation subspace for both $x$ and $y$. For fixed approximate solution $u_{h} \in X_{h}$, since $\Delta^{2} u_{h} \in X_{h}$, we can apply direct residual formulation in Section 1.2.1 such that

$$
\left\{\begin{aligned}
P \Delta^{2} w & =J\left(w+u_{h}, \Delta w\right)+J\left(w, \Delta u_{h}\right)-P \Delta^{2} u_{h}+\sqrt{P R} \xi+J\left(u_{h}, \Delta u_{h}\right) & \text { in } \quad \Omega \\
w & =\Delta w=0, & \text { on } \quad \partial \Omega
\end{aligned}\right.
$$

for

$$
w:=u-u_{h}, \quad J(u, v):=u_{x} v_{y}-v_{x} u_{y} .
$$

Verification algorithm IS-Res requires a maximum norm estimation by Plum [12] such that

$$
\|v\|_{L^{\infty}(\Omega)} \leq K_{1}\|v\|_{L^{2}(\Omega)}+K_{2}\|\nabla v\|_{L^{2}(\Omega)}+K_{3}\|\Delta v\|_{L^{2}(\Omega)}, \quad \forall v \in X
$$

where

$$
K_{1}=\frac{1}{\pi} \sqrt{\frac{a}{2}}, \quad K_{2}=1.1548 \sqrt{\frac{4+a^{2}}{6 a}}, \quad K_{3}=0.22361 \sqrt{\frac{144+40 a^{2}+9 a^{4}}{10 a^{3}}} \frac{\pi}{3} .
$$

By using Eq. (22) and similar norm estimations described in [22], it can be checked

$$
\left\|v_{x}\right\|_{L^{\infty}(\Omega)} \leq C_{1}\|v\|_{X}, \quad\left\|v_{y}\right\|_{L^{\infty}(\Omega)} \leq C_{2}\|v\|_{X}, \quad \forall v \in X
$$

with

$$
C_{1}:=(2 \sqrt{3} / 9) K_{1}+(1 / 2) K_{2}+K_{3}, \quad C_{2}:=\left(2 \sqrt{3} /\left(9 a^{2}\right)\right) K_{1}+(1 /(2 a)) K_{2}+K_{3} .
$$

Thus using Hölder's inequality and the triangle inequality, we have

$$
\sup _{w \in W}\|g(w)\|_{Y} \leq \alpha\left(C_{1}\left(\alpha+t_{3}\right)+C_{2}\left(\alpha+t_{2}\right)+t_{4}\right)+t_{1}
$$

where

$$
\begin{gathered}
t_{1}:=\left\|-P \Delta^{2} u_{h}+\sqrt{P R} \xi+J\left(u_{h}, \Delta u_{h}\right)\right\|_{L^{2}(\Omega)}, \\
t_{2}:=\left\|\left(\Delta u_{h}\right)_{x}\right\|_{L^{2}(\Omega)}, \quad t_{3}:=\left\|\left(\Delta u_{h}\right)_{y}\right\|_{L^{2}(\Omega)}, \quad t_{4}:=\left\|\left(u_{h}\right)_{x}\right\|_{L^{\infty}(\Omega)}+\left\|\left(u_{h}\right)_{y}\right\|_{L^{\infty}(\Omega)} .
\end{gathered}
$$

Figure 3 shows the shape of the approximate solution. For $R=5, P=10, a=1 / \sqrt{2}, N=5$, IS-Res succeeded to enclose the solution within $3.67713 \times 10^{-9}$ by meaning of $\|W\|_{X}$. We can not assure the local uniqueness of the solution. In order to apply to larger $R$ or the Oberbeck-Boussinesq equation, IS-Res has the limitation. Therefore, other verification algorithms, for example, based on Newton method [22] will be useful.

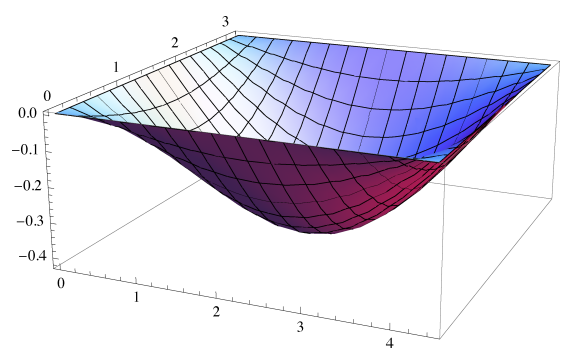

Fig. 3. Shape of approximate solution. 


\section{Acknowledgments}

The author heartily thanks the anonymous referee for his/her thorough reading and valuable comments. This work was supported by a Grant-in-Aid from the Ministry of Education, Culture, Sports, Science and Technology of Japan (No.20224001, No.21540134).

\section{References}

[1] M.T. Nakao, "Solving nonlinear elliptic problems with result verification using an $H^{-1}$ type residual iteration," in Validation Numerics: theory and applications, eds. R. Albrecht, G. Alefeld, and H.J. Stetter, pp. 161-173, Computing Supplementum, vol. 9, Springer-Verlag, Wien, 1993.

[2] P.G. Ciarlet, The Finite Element Method for Elliptic Problems, North-Holland, Amsterdam, 1978.

[3] S.M. Rump, "Verification methods: Rigorous results using floating-point arithmetic," Acta Numerica, vol. 19, pp. 287-449, May 2010.

[4] S.M. Rump, "INTLAB - INTerval LABoratory," in Developments in Reliable Computing, ed. T. Csendes, pp. 77-104, Kluwer Academic Publishers, Dordrecht, 1999. http://www.ti3.tu-harburg.de/rump/

[5] N. Yamamoto and M.T. Nakao, "Numerical verifications for solutions to elliptic equations using residual iterations with a higher order finite element," Journal of Computational and Applied Mathematics, vol. 60, no. 1-2, pp. 271-279, June 1995.

[6] M.T. Nakao and N. Yamamoto, "Numerical verification of solutions for nonlinear elliptic problems using $L^{\infty}$ residual method," Journal of Mathematical Analysis and Applications, vol. 217, no. 1, pp. 246-262, January 1998.

[7] N. Yamamoto and M.T. Nakao, "Numerical verifications of solutions for elliptic equations in nonconvex polygonal domains," Numerische Mathematik, vol. 65, no. 1, pp. 503-521, December 1993.

[8] E. Zeidler (Translated by P.R. Wadsack), Nonlinear Functional Analysis and its Applications, Part I: Fixed-Point Theorems, Springer-Verlag, 1986.

[9] N. Yamamoto, "A numerical verification method for solutions of boundary value problems with local uniqueness by Banach's fixed point theorem," SIAM Journal on Numerical Analysis, vol. 35, no. 5, pp. 2004-2013, October 1998.

[10] S.M. Rump, "A note on epsilon-inflation," Reliable Computing, vol. 4, no. 4, pp. 371-375, November 1998.

[11] S. Oishi, "Numerical verification of existence and inclusion of solutions for nonlinear operator equations," Journal of Computational and Applied Mathematics, vol. 60, no. 1-2, pp. 171-185, June 1995.

[12] M. Plum, "Explicit $H_{2}$-estimates and pointwise bounds for solutions of second-order elliptic boundary value problems," Journal of Mathematical Analysis and Applications, vol. 165, no. 1, pp. 36-61, March 1992.

[13] J.G. Heywood, W. Nagata, and W. Xie, "A numerically based existence theorem for the NavierStokes equations," Journal of Mathematical Fluid Mechanics, vol. 1, no. 1, pp. 5-23, April 1999.

[14] M.T. Nakao and Y. Watanabe, "Numerical verification methods for solutions of semilinear elliptic boundary value problems," NOLTA, vol. 2, no. 1, pp. 2-31, January 2011.

[15] M.T. Nakao, "A numerical approach to the proof of existence of solutions for elliptic problems II," Japan Journal of Applied Mathematics, vol. 7, no. 3, pp. 477-488, October 1990.

[16] M.T. Nakao and Y. Watanabe, Self-validating Numerical Computations by Learning from Examples: Theory and Implementation, The Library for Senior \& Graduate Courses 85, Saiensu-sha (in Japanese).

[17] M.T. Nakao, N. Yamamoto, and S. Kimura, "On best constant in the error bound for the $H_{0^{-}}^{1}$ projection into piecewise polynomial spaces," Journal of Approximation Theory, vol. 93, no. 3, 
pp. 491-500, June 1998.

[18] M.H. Schultz, Spline Analysis, Prentice-Hall, London, 1973.

[19] P.L. Lions, "On the existence of positive solutions of semilinear elliptic equations," SIAM Review, vol. 24, no. 4, pp. 441-467, October 1982.

[20] F. Kikuchi and X. Liu, "Determination of the Babuska-Aziz constant for the linear triangular finite element," Japan Journal of Industrial and Applied Mathematics, vol. 23, no. 1, pp. 75-82, February 2006.

[21] P. Grisvard, Elliptic Problems in Nonsmooth Domains, Pitman, Boston, 1985.

[22] Y. Watanabe, N. Yamamoto, M.T. Nakao, and T. Nishida, "A numerical verification of nontrivial solutions for the heat convection problem," Journal of Mathematical Fluid Mechanics, vol. 6, no. 1, pp. 1-20, March 2004. 